\title{
Giant vulva fibroma presenting as a genital mass: a case report
}

\author{
Edgar Ndaboine ${ }^{1,2}$, Dismas Matovelo ${ }^{1 *}$, Arnold Itemba $^{1}$, Cosmas Mbulwa ${ }^{2}$ \\ ${ }^{1}$ Catholic University of Health and Allied Sciences (CUHAS), Department of Obstetrics \& Gynecology, P.O.BOX 1464, \\ Mwanza, Tanzania, \\ ${ }^{2}$ Bugando Medical Centre (BMC), Department of Histopathology, P.O.BOX 1370, Mwanza, Tanzania
}

\begin{abstract}
Introduction: Even though vulva fibroma is rare, it is among the solid tumors of the vulva. Its cause is unknown although it has been associated with physiological hormonal changes. We report a patient with a unique vulva fibroma which has grown to the extent of interfering with her gait and urination.

Case presentation: A 22-year-old woman presented with a genital mass which had been present for the duration of 2 years and felt embarrassed to report to hospital early as the growth was in the genital area, with perception of it being a sexually related illness, despite having not yet started engaging in sexual activity. On physical examination, a palpable pendulous mass of about [30 $\mathrm{x} 22] \mathrm{cm}$ was seen originating from right labia and extending to the right perineum. The mass was firm, nodulated, non-tender and had limited mobility. Surgical excision was performed under spinal anesthesia. Histologically, features suggestive of mixoid fibroma were reached after the mass was excised. No recurrence has been observed.

Conclusion: Clinicians should be aware of this rare disease which can be associated with recurrence if there is incomplete excision. Again, the unusual finding of the genital mass can be very embarrassing to the patient affecting her psychosocial well-being. It needs to be treated immediately upon diagnosis.
\end{abstract}

Keywords: Vulva fibroma, vulval mass, excision, a case report

\section{Background:}

Vulva Fibroma is a rare benign vulva tumor, it has been described previously in prepubertal period and very few reported cases during pubertal and postmenopausal period(Makhloufi W 2016). It is due to overgrowth of the normal stromal tissues of the vulva as a result of physiological pubertal hormonal changes (Zhang, J et al. 2011). It may be congenital or acquired type with unknown cause. Approximately $30 \%$ of cases are associated with recurrence because of incomplete excision of the tumor (Ajibona, $\mathrm{O}$ et al. 2007). Here we report a case of a giant vulva fibroma in a 22-year-old woman who was not sexually active and presenting at a young age.

\section{Case Presentation:}

A 22-year-old female, nulliparous, single, living with parents from the northwestern region of Tanzania presented at Bugando Medical Centre (BMC) outpatient clinic in Mwanza city with complaints of genital swelling for the duration of 2 years. The mass started as a pimple- like structure, increasing in size gradually with time. The swelling grew up to the point of covering the whole genital part. Initially there was no pain but later she experienced discomfort, pain when walking, and difficulties in urinating.

She felt embarrassment to report to hospital early as the growth was in genital area, with perception of being a sexually related illness, even though she had not yet started engaging in sexual activity. She was only taking self-prescribed pain killers until she started to have difficulty urinating and her unusual gait was spotted by her mother which led to disclosure and hospital care attention. She attained menarche at 16 years with regular menstrual cycle of 28 days, 3-4 days per cycle with light flow changing 3 pads per day.

*Corresponding E-mail: magonza77@yahoo.co.uk 
On physical examination, she appeared to be healthy, with no lower limb edema, not dyspneic, not jaundiced, not cyanosed, afebrile at $36.6{ }^{\circ} \mathrm{C}$ with blood pressure of $110 / 75 \mathrm{mmHg}$. On pelvic examination, a palpable pendulous mass of about [30 $\times 22] \mathrm{cm}$ was seen (Figure 1).

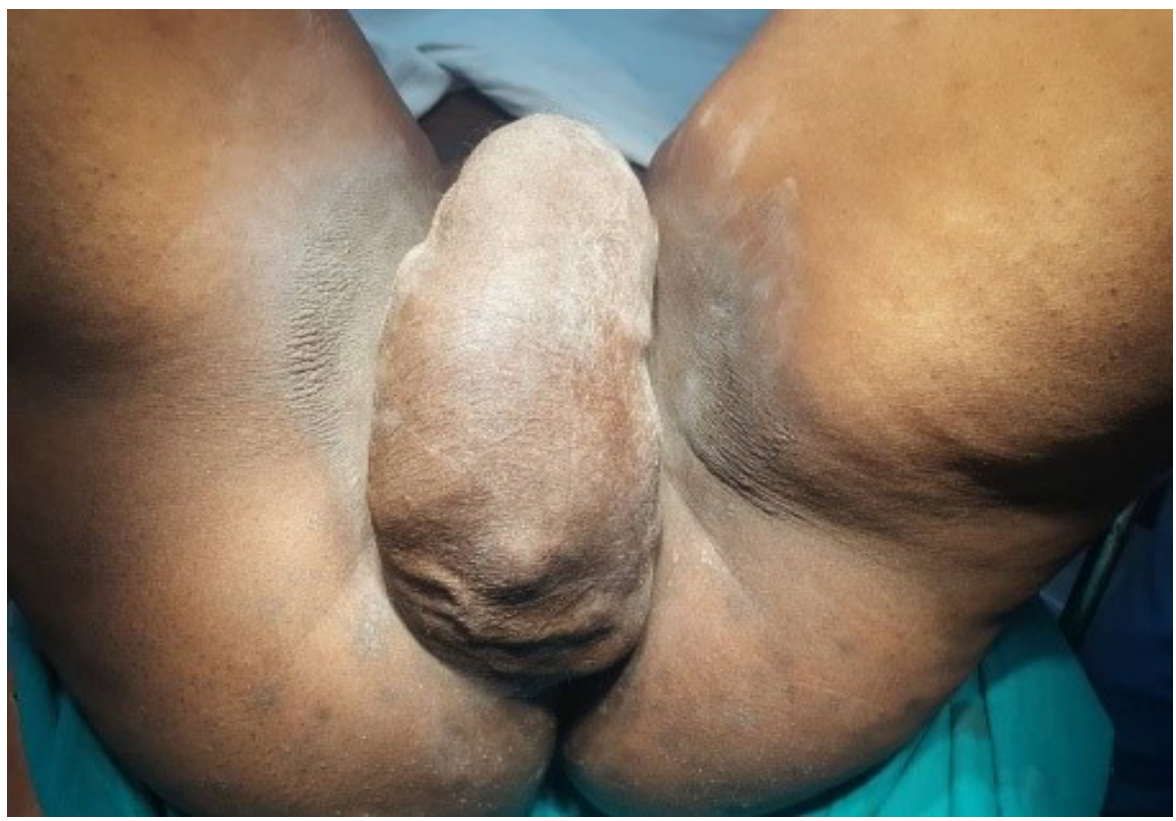

Figure 1: A pendulous mass of about [30 x 22] cm as identified during physical examination.

The mass was originating from right labia extending to the right perineum. The mass was firm, nodulated, non-tender and with limited mobility. She had no enlarged inguinal lymph nodes. The digital vaginal examination was not performed as she was not sexually active. Patient was counseled for surgical excision to which she consented.

\section{Treatment}

In the operating theatre under spinal anesthesia; a huge right solid vulva tumor measuring $30 \times 22 \mathrm{~cm}$ in length was identified originating from right labia, mobile, not fixed to skin, perineal muscles or fascia. It was surrounded with multiple varicose veins. The clitoris, urethra, and vagina mucosa were normal although compressed and pushed to the left side. Inguinal lymph nodes were not palpable.

Surgical excision in totality of the tumor was carried out successfully by using diathermy for separation of skin and adjacent structures. Care was also taken to minimize bleeding by identification of blood vessels and proper ligation. The wound was then closed without leaving any drain in place. The sample was then sent for histological examination. At the pathology department, an encapsulated huge greyish mass of $17 \times 11 \times 10 \mathrm{~cm}$ was identified. A cut surface was greyish throughout the cut which looked like a myoma (Figure 2). 


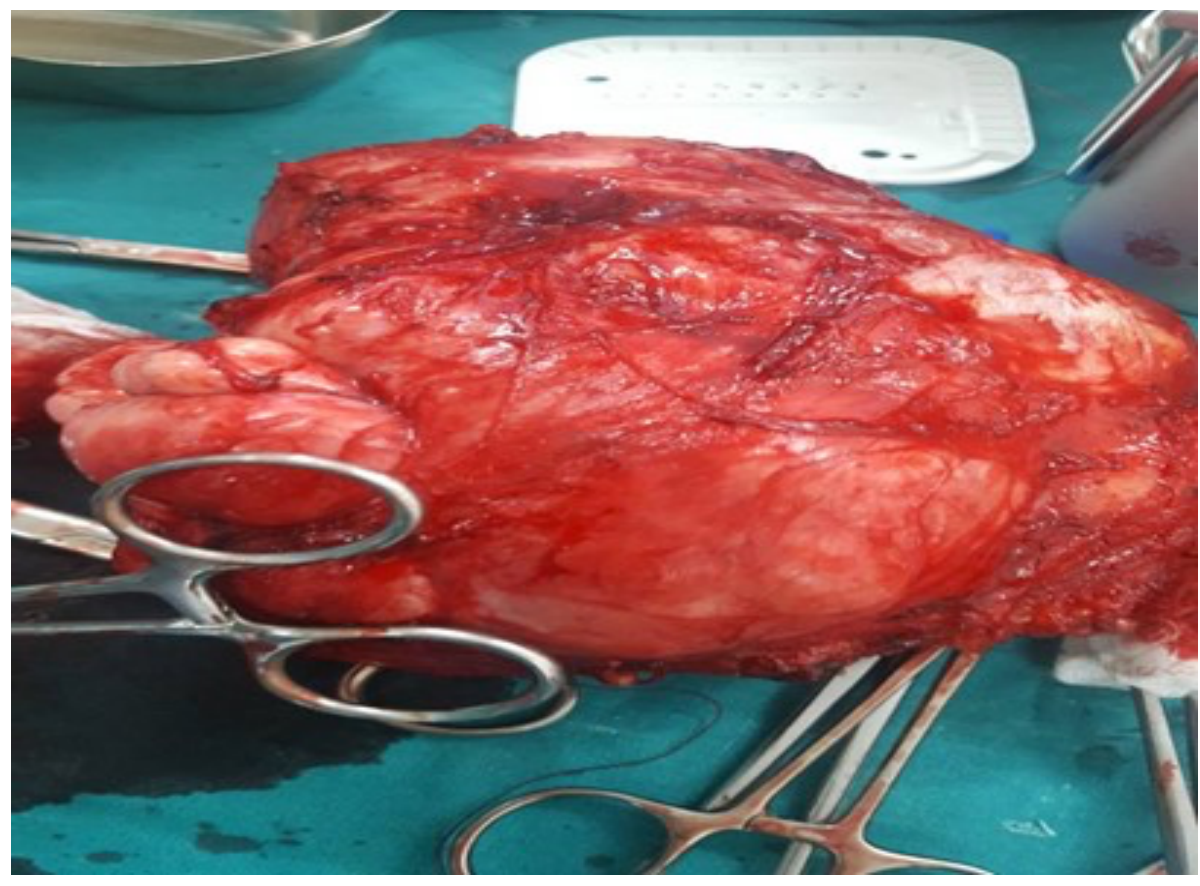

Figure 2: An encapsulated greyish mass of 17 x 11 x $10 \mathrm{~cm}$ was excised.

Outcome She had uneventful post-operative recovery with excellent cosmetic result as indicated in the image below (Figure 3).

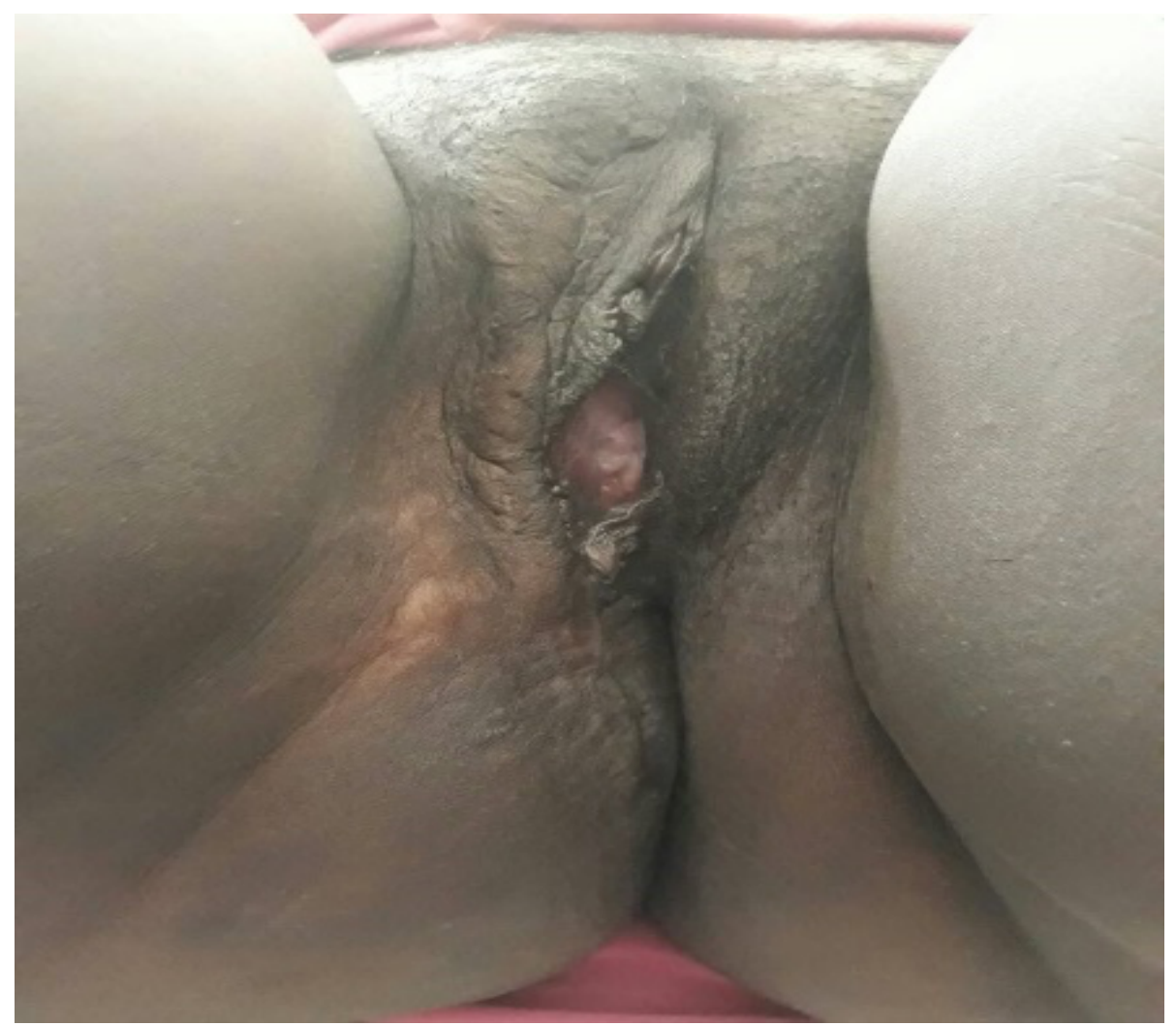

Figure 3: A post-operative view of the external genitalia. 
The tissue sample was selected, and sections were stained by Hematoxylin and Eosin (H\&E) and observed by a light microscopy. Histology revealed a fibroblast and mixoid changes without atypia. Diagnosis of mixoid fibroma was made (Figure. 4).

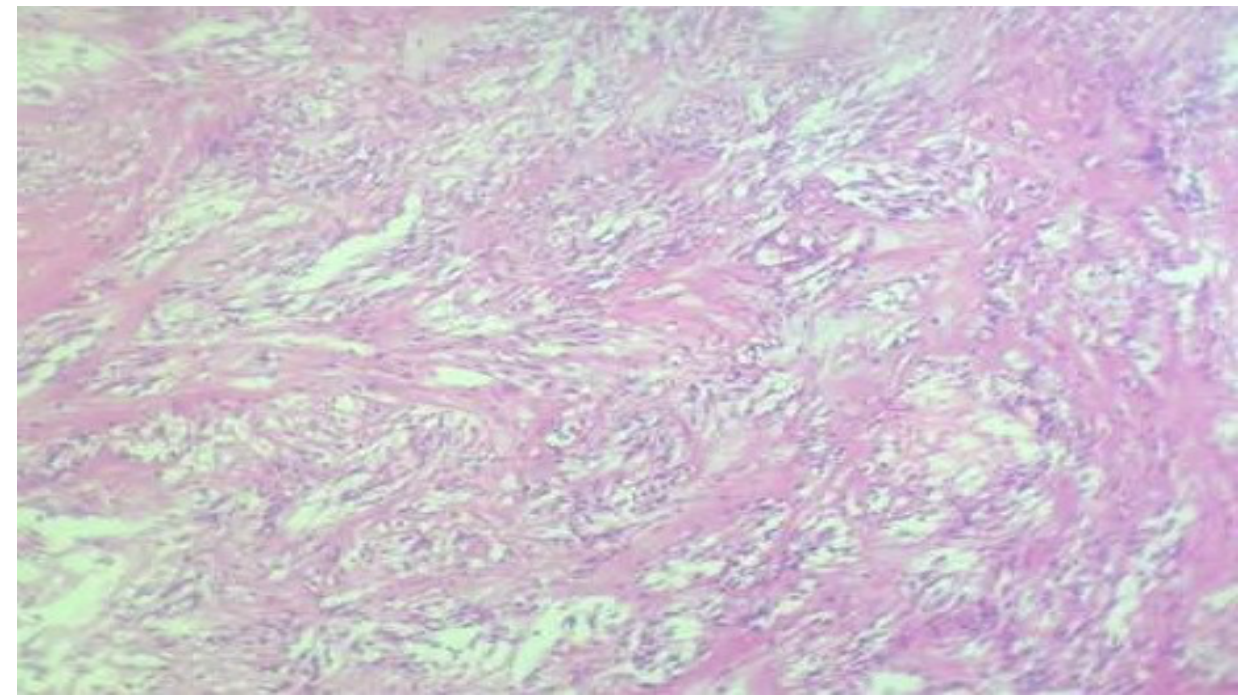

Figure 4: $\mathrm{H} \& \mathrm{E}$ histology section (X40 power) showing areas of fibroblast, mixoid changes and neovascularization.

\section{Follow-up}

The patient reported to be delighted to have her gait and urination returned to normal. Again, she had not shown any sign of disease recurrence for 12 months following hospital discharge. She was still on biannual surveillance.

\section{Discussion}

Vulva fibroma is a sporadic benign tumor of the vulva and very few are described in the literature with estimated incidence of $0.03 \%$. Most of them are detected much earlier and are treated by surgical excision (Chen, D et al. 2004, Makhloufi W 2016). To our awareness this will be the first giant vulva fibroma to be reported in Mwanza, Tanzania. Fibromas may perhaps arise from deep connective tissues of fibroblasts, with a diffusely myxoid stroma, slight collagen fibrils and perivascular smooth muscle proliferation. Lesions are predominantly found on the labium, and range in different sizes from small pimple like to giant in size (Hoofman, BL et al. 2008, Najam, R et al. 2013).

In this case, the patient noticed a very small growth in her genitals which increased progressively to giant size causing abnormal gait and difficulties in urination due to compression. She used to push and hold the tumor during urination for better and easy flow of urine. Initial lack of pain, fear and embarrassment have been identified to be major barriers for early seeking of medical care that ultimately lead to development of giant vulva fibroma (Hoofman, BL et al. 2008). Our patient also delayed seeking medical attention for more than 2 years due to similar reasons. Another reason was being from a conservative family which does not talk much on sexuality, which created a fear of being judged and of being involved in sexual activity before marriage. This raises an importance matter related to ability to discuss sexuality matters at the family level which will ultimately help improve early care seeking behaviour.

In literature there are rare reports of having similar giant fibroma for 7 to 30 years of existence without seeking medical help (Ajibona, O et al. 2007, Abasiattai, AM et al. 2010, Najam, R et al. 2013). Fibroma commonly occurs at reproductive age, however, there are also reports in infants and postmenopausal women (Iwasa and Christopher 2004, Hoofman, BL et al. 2008). Important 
differential diagnosis includes Lipoma, leiomyoma, inguinal hernia, vulvovaginal cysts, vulval elephantiasis, and fibro epithelioid tumours (Chen, D et al. 2004, Hoofman, BL et al. 2008, Makhloufi W 2016). Complete surgical excision is the only curative treatment of choice to date with minimal recurrence for incomplete excision. Our limitation in this case was that special immunostaining were not performed due to unavailability at our facility at the time this case was managed.

\section{Conclusion}

The unusual finding of the genital mass can be very embarrassing to the patient affecting her psychosocial well-being. It needs to be treated immediately upon diagnosis.

Ethics approval and consent to participate: Not applicable, however, consent was taken from the patients for carrying out this work.

Consent to publish: A written informed consent was obtained from the patient for publication of this case report. A copy of the written consent is available for review by the Editor-in- Chief of this journal.

Availability of data and materials: All data analyzed during the study are included in this published article

Competing Interests: Authors declare that they have no competing interests.

Funding: No funding was obtained for this study

Authors Contributions: EN reviewed the patient prior to surgery, performed the surgery and reviewed several drafts of the manuscript. DM wrote the initial draft of manuscript and revised several drafts of the manuscript. Al did a follow-up of the patient prior to surgery, assisted the surgery, followed up the patient after surgery, histological results and reviewed the manuscripts. CM did all the histological studies and later reviewed the final draft of the manuscript. All authors read and approved the final manuscript.

Acknowledgments: The authors acknowledge help from operating theatre and gynecological ward personnel who assisted in the care of this patient throughout her stay in our hospital.

\section{References}

Abasiattai, et al. (2010). "Rare disease: Giant fibroma of the vulva." BMJ case reports 2010. doi: $10.1136 / \mathrm{bcr} .05 .2010 .2985$

Ajibona, et al. (2007). "A distinctive vulval fibroma of so-called prepubertal type in a postmenopausal patient." J Clin Pathol 60(4): 437-438. DOI:10.1136/jcp.2006.040428

Chen, et al. (2004). "Huge pedunculated fibroma of the vulva." Acta obstetricia et gynecologica Scandinavica 83(11): 1091-1092. https://doi.org/10.1111/j.0001-6349.2004.0122b.x

Hoofman, et al. (2008). "Benign Disorders of the Lower Reproductive tract." Williams Gynecology Chapter 4: McGraw Hill Professional. https://accessmedicine.mhmedical.com/Content. aspx?bookld=399\&sectionld=41722292

Iwasa and Christopher (2004). "Distinctive prepubertal vulval fibroma: a hitherto unrecognized mesenchymal tumor of prepubertal girls: analysis of 11 cases." The American journal of surgical pathology 28(12): 16011608. DOI:10.1097/00000478-200412000-00008

Makhloufi W., Sayah C., Choutri H (2016). "Giant Soft Fibroma Located in Labia Majora: Pediatric Case Report." Gynecol Obstet Case Rep 2: 3. DOI: 10.21767/2471-8165.1000034 
Najam, et al. (2013). "A Large Fibroma Polyp of Labia Majora-A Case Report." J Clin Case Rep 3(297): 2. doi.org/10.4172/2165-7920.1000297

Zhang, et al. (2011). "Prepubertal vulval fibroma with a coincidental ectopic breast fibroadenoma: report of an unusual case with literature review." Journal of Obstetrics and Gynaecology Research 37(11): 1720-1725. doi: 10.1111/j.1447-0756.2011.01580 